\title{
Detection and Recognition of Characters in Place Name Board for Driving
}

\author{
S.Sathiya \\ Assistant Professor \\ Department of Computer Science And Engineering \\ Annamalai University
}

\author{
M.Balasubramanian \\ Assistant Professor \\ Department of Computer Science And Engineering \\ Annamalai University
}

\author{
S. Palanivel \\ Professor \\ Dept. of CSE \\ Annamalai University
}

\begin{abstract}
The objective of this paper is to detect and recognize the characters in the signage's (traffic place name board). It is applicable especially for Indian conditions. It detect the green colored signage's from the background using seamearing algorithm and extract the detected signage's board then convert it into binary image. Next, it detect the signage characters from signage's using horizontal segmentation and vertical segmentation algorithms, it extract each individual character from the signage's. Then, the features are extracted from individual character of signage's using DCT, DWT and Hybrid DWT-DCT. In training phase, 324 discrete wavelet features are extracted from 36 characters $(9$ features extracted from 36 character) in DWT, 20 highest energy coefficients are extracted by using DCT and 20 highest energy coefficients are extracted using Hybrid DWT-DCT. Finally, the extracted features from each characters are recognized using SVM. Selection of feature is probably important factor to achieve high performance in recognition. The application of this paper is a driver assistant system, to guide the driver while driving, traffic safety by calling the driver's attention to the presence of key traffic information board. The performance of signage recognition is evaluated for place board image and the system achieves a recognition rate of $94.44 \%$ using DWT , 91.66 $\%$ using DCT, $97.22 \%$ using Hybrid DWT-DCT and SVM.
\end{abstract}

\section{Keywords:}

Discrete Cosine Transform(DCT), Discrete Wavelet Transform(DWT), Feature Extraction, Support Vector Machine(SVM)

\section{INTRODUCTION}

Image texts carry useful information to the people. The captured texts are in different sizes, different variations and it consider like an image then transferring it into machine understandable form. Text detection is to extract the region of text alone to identify each character and numbers through its machine known format. Still images are taken as an input. The acquired images are taken as input in the format of BMP format. The resolution of the captured image is $320 * 240$ in RGB format, and it were taken from approximately 3-5 meters away from the signage board.

\subsection{Related Work}

Several approaches have been proposed in the literature for stillimage based signage text detection and recognition such as robust text detection, wavelet and gabor for feature extraction used to localize the text properly [1]. The detected text directly fed into the OCR recognition [2]. Several methods have been proposed, to recognize the text using SVM, MRF, video OCR [3]. For still-image based and video frame based detection and the recognition of text in a complicated background like buildings, trees, animals, pedestrians and different vehicles . For text detection edge feature and morphology operation to locate the edge dense image block. Wavelet used to extract the feature and SVM used to recognize text [4]. This work describes the localization and segment the text in stillimages and videos using gradient feature and complex valued multilayer feed forward network classifier [5]. To localize the text under complicated background using gabor filter and SVM used as recognizer[6]. Directional pattern matching used to recognize the text[?]. To segment the text using latent dirichlet allocation(LDA), multinomial mixture[9]. we explore a new framework to detect text strings with arbitrary orientations in complex natural scene image. The text line grouping method performs Hough transform to fit text line among the centroids of text candidates. Each fitted text line describes the orientation of a potential text string[10]. We propose a novel algorithm of scene text detection and localization[11]. Automatically segmentation of text in different probabilistic models using HMM [12]. In citem, tutorial on support vector machine for pattern recognition were proposed. The proposed hybrid DWT-DCT algorithm performs much better than the DCT, DWT. This scheme reduces false contouring and blocking artifacts significantly. The rate distortion analysis shows that for a fixed level of distortion, the number of bits required to transmit the hybrid coefficients would be less than those required for other schemes[14]and[15]. 


\subsection{Outline of Work}

The still image based characters recognition system from signage's are described in this paper consist of four modules. Detection and extraction of signage's, segmentation of characters, character feature extraction and reorganization of characters using SVM. Detection and extraction of signage's are described in section 2 Segmentation of characters are described in section 3 Character feature extractions are described in section 4 Recognition of characters using SVM are described in section 5 Experimental results are described in section 6 and Conclusion in section 7 Block diagram of proposed work is shown in figure 1

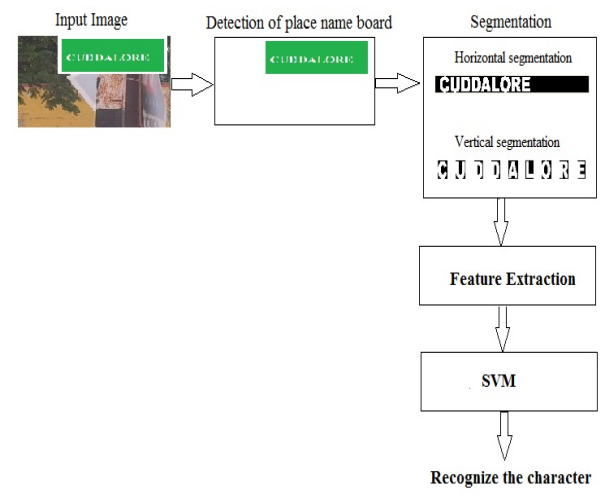

Fig. 1. Block diagram of proposed work

\section{DETECTION AND EXTRACTION OF SIGNAGE'S}

To detect the traffic place name board (signage) from the background using color information. Color image are represented by using RGB color space. Each pixel can be identified using Red component, Green component and Blue component. The detection of the board using this color specification.

\subsection{Signage region extraction}

Smearing algorithm is used to extract the place board in an image. The smearing algorithm is searching for the first and last green pixels starting from top left corner of an image, it can extract only green pixels from the given image. The image is then automatically segmented to show only place name board. The grouping region is separately filled components. In order to do the bounding boxes for each region was computed. By choosing the maximum area of bounding boxes, in which the place name board location was found. The place board must be free of alignment errors.

\section{PLACE BOARD CHARACTER SEGMENTATION}

The extracted place name board is converted into binary image, like black colored background and white colored letters. Horizontal segmentation is used as line separation, the line that contain full black are removed (Horizontal line). Likewise Vertical segmentation approach is to remove the unwanted column wise black pixels(vertical line). It segment the individual character to identify each character in machine understandable form. In horizontal segmentation approach, count the pixel in row wise from starting point of the board to ending point board if the counting value is 0 means then leave that row and go to another row this process continues up to end of the board, else the count value is greater than 255 means then consider that line, that contains text then extract that line. We get the row segmented character, which is shown in Figure 2

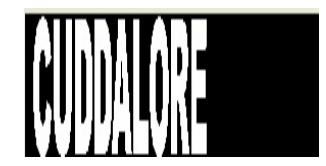

Fig. 2. Line separation using horizontal segmentation.

In vertical segmentation approach, count the pixel in column wise from starting point of the top board to ending point board, if the count value is 0 means then leave that column and go to another column this process continues up to end of the column. If count value is greater than 255 means, then consider that particular line, that contains text and restore that column. It eliminate the unwanted space before the column and after the columns finally character are square in shape. Each square having the segmented character to identify individually, and recognize it. The individual segmented characters are shown in Figure 3.

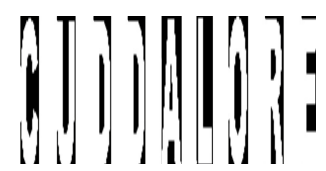

Fig. 3. Character separation using vertical segmentation.

\section{CHARACTER FEATURE EXTRACTION}

Important object representation components are extracted by DCT and DWT feature extraction techniques. It reduces the features for computation. The reduced features to represent the object in a efficient manner. It can be done by using two techniques, DCT, DWT and Hybrid DWT-DCT.

\subsection{Feature Extraction using DCT}

Discrete cosine transform (DCT) is a feature extraction technique proposed for segmented characters. It is a powerful transform to extract more important features for characters recognition. DCT is applied to all segmented characters and numbers from 0 to 9 images (36 images), important features of the coefficients are selected to represent feature vectors in a zigzag manner or by zonal masking.

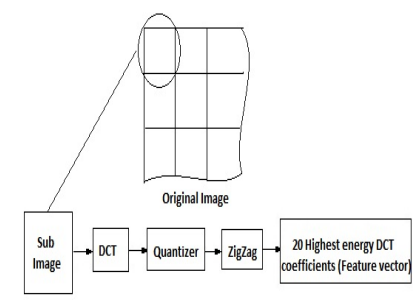

Fig. 4. DCT is performed on each block.

Figure 4 is to perform DCT on each block. To perform the feature reduction, an image is first subdivided into blocks of $8 \times 8$ pixels. 
The DCT is then performed on each block. This generates 64 coefficients which are then quantized to reduce their magnitude. The coefficients are then reordered into a one-dimensional array in a zigzag manner before further entropy encoding. To keep consistency with matrix notation, pixel locations (and image sizes) are described using row(s) first, then column(s). Here segmented individual characters given as input image block are given as $\mathrm{f}(\mathrm{y}, \mathrm{x})$, where $\mathrm{y}, \mathrm{x}=0,1,2 . \mathrm{N}-1$, we decompose it into 2D DCT basis functions. The result is an NXN matrix $\mathrm{C}(\mathrm{v}, \mathrm{u})$ containing DCT coefficient in equation (1)and (2).

$c(v, u)=\alpha(v) \alpha(u) \sum_{y=0}^{N-1} \sum_{x=0}^{N-1} f(y, x) \cos \left[\frac{(2 y+1) v \pi}{2 N}\right] \cos \left[\frac{(2 x+1) u \pi}{2 N}\right]$

For $\mathrm{v}, \mathrm{u}=0,1,2, ., \mathrm{N}-1$,

$$
\alpha(v)=\sqrt{\frac{1}{N}} \text { for } \mathrm{v}=0, \text { and } \alpha(v)=\sqrt{\frac{2}{N}} \text { for } \mathrm{v}=1,2, \ldots . \mathrm{N}-1
$$

The coefficients are ordered according to Zigzag pattern, it is reflecting the amount of information stored. For block located at $(\mathrm{b}, \mathrm{a})$, the DCT vector is composed of, $\left[c_{c}\right]$ in equation 3 .

$$
X=\left[c_{0}^{(b, a)} c_{1}^{(b, a)} \ldots . . c_{(M-1)}^{(b, a)}\right]^{T}
$$

Where $\mathrm{M}$ is the number of retained coefficients. That is, $\mathrm{M}=20$ and $\left.c_{n}^{(} b, a\right)$ denotes the nth DCT coefficients. After applying $D C T$, 20 highest energy coefficients are extracted from each segmented image. First coefficient is a $D C$ coefficient and the remaining 19 coefficients are $A C$ coefficients.

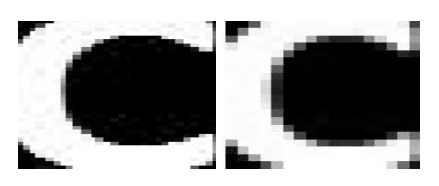

Fig. 5. DCT Conversion for Character 'C' Original Image and DCT Image.

Table 1. 20 Highest Energy Coefficients of DCT

$1.00920 .5736-0.05490 .48200 .02530 .15560 .22400 .0892-0.16680 .0321$ $0.11890 .0193-0.46880 .0207-0.1625-0.0430-0.0044-0.10560 .0317-0.1315$

\subsection{Feature Extraction using DWT}

DWT is a powerful transform to extract important feature from the given set of data. For many applications, the low-frequency coefficients are the more important one. It is what gives the identity. In wavelet analysis, we often think of approximations and details. The approximations are the high-scale, low-frequency components of the signal. The details are the low-scale, high-frequency components. The DWT of a signal is calculated by passing it through a series of filters. First the samples are passed through a low pass filter with impulse response resulting in a convolution of the two in equation 4:

$$
y[n]=(x * g)[n]=\sum_{k=-\infty}^{\infty} x[k] g[n-k]
$$

The signal is also decomposed simultaneously using a high pass filter. The outputs giving the detail coefficients (from the high-pass filter) and approximation coefficients (from the low-pass). It is important that the two filters are related to each other and they are known as a quadrature mirror filter.. However, since half the frequencies of the signal have now been removed, half the samples can be discarded according to Nyquist's rule. The filter outputs are then subsample into 2 (Mallet's and the common notation is the opposite, $\mathrm{g}$ - high pass and $\mathrm{h}$ - low pass) in equation 5 and 6 :

$$
\begin{aligned}
& y_{\text {low }[n]}=\sum_{k=-\infty}^{\infty} x[k] g[2 n-k] \\
& y_{h i g h[n]}=\sum_{k=-\infty}^{\infty} x[k] g[2 n-k]
\end{aligned}
$$

This decomposition has halved the time resolution since only half of each filter output characterizes the signal. However, each output has half the frequency band of the input so the frequency resolution has been doubled.

$$
(y \downarrow k)[n]=y[k n]
$$

Equation (7) is the summation can be written more concisely.

$$
\begin{gathered}
y_{\text {low }}=(x * g) \downarrow 2 \\
y_{\text {high }}=(x * h) \downarrow 2
\end{gathered}
$$

However computing a complete convolution with subsequent down sampling in equation 8 and 9 would waste computation time. The Lifting scheme is an optimization where these two computations are interleaved.

4.2.1 Cascading and Filter banks. This decomposition is repeated to further increase the frequency resolution and the approximation coefficients decomposed with high and low pass filters and then down-sampled. This is represented as a binary tree with nodes representing a sub-space with a different time-frequency localization. The tree is known as a filter bank.

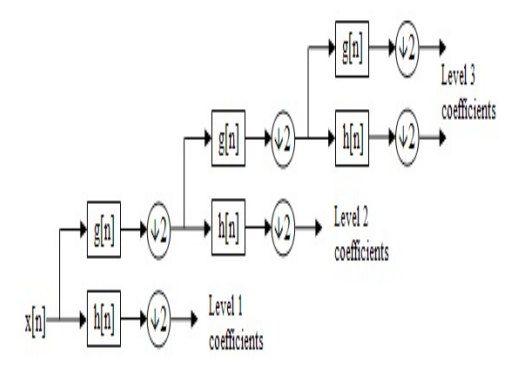

Fig. 6. A Three level filter bank.

At each level in the Figure 6 the signal is decomposed into low and high frequencies. Due to the decomposition process the input signal must be a multiple number of levels. For example a signal with 32 samples, frequency range 0 to $f_{n}$ and three levels of decomposition, 4 output scales are produced. Figure 7 is the frequency representation of DWT. 
1

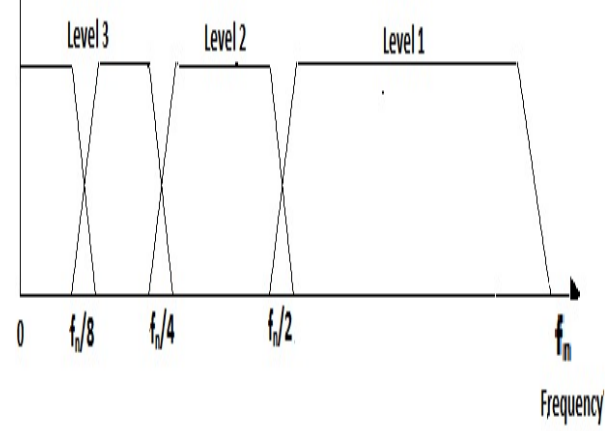

Fig. 7. 3 Frequency domain representation of the DWT.

4.2.2 DWT Coefficients. The input signal $\mathrm{x}(\mathrm{n})$ is decomposed into two sets of coefficients called approximation coefficients (denoted by $C A$ ) and detail coefficients (denoted by $C D$ ). These coefficients are obtained by convolving the input signal with a low-pass filter (for $C A$ ) or a high-pass filter (for $C D$ ) and then down sampling the convolution result by 2 . The size of $C A$ and $C D$ is half of the size of the input signal for Periodic extension mode, and half of the sum of the input signal's size and the filter's size for Zeropadded extension mode. Note that the filters are determined by the chosen wavelet.

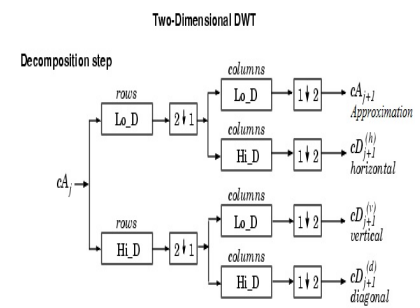

Fig. 8. Two dimensional wavelet decomposition.

The filtering or decomposition process is shown in Figure 8 LoDCA and HiD are lowpass and high pass decomposition filters, respectively. $2 \downarrow 1$ or $1 \downarrow 2$ represents down sampling by 2 . $C D$ and $C A$ are the approximation and detail coefficients.

4.2.3 Daubechies wavelet. Daubechies wavelet is based on the use of recurrence relations to generate progressively finer discrete samplings of an implicit mother wavelet function; each resolution is twice that of the previous scale. Segmented characters are used to extract feature, segmented characters for feature extraction are shown in Figure 9

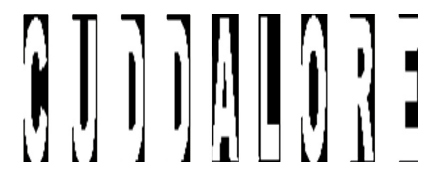

Fig. 9. Segmented character for feature extraction.

The features are extracted from individual segmented character of place board. In training phase, 324 discrete wavelet features are extracted from 36 characters. In each character 9 feature are extracted from 36 character). Figure 10 shows first level of decomposition of segmented character ' $C$ '. The individual objects are passed through the filter, which contains the four partitions,

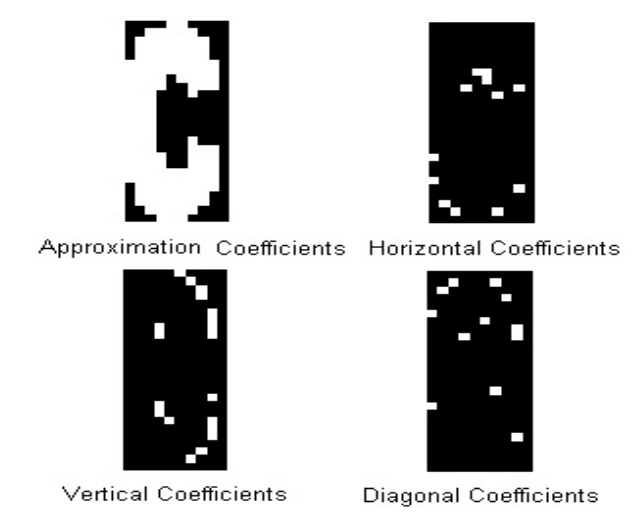

Fig. 10. First level of decomposition of segmented character 'C'.

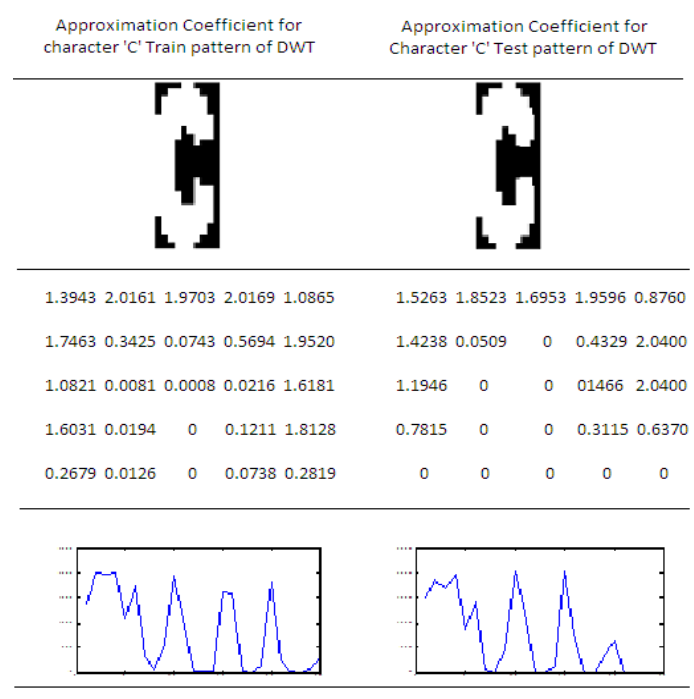

Fig. 11. Train and Test pattern of DWT

\subsection{Feature Extraction using Hybrid DWT-DCT}

\section{CHARACTER RECOGNITION USING SVM}

Support vectors machine[13] and [14] is based on the principle of Structural Risk Minimization(SRM). It is classification model. Support vector are used to find hyper plane between two classes. Support vectors are the training samples that define the optimal separating hyper plane. Support vectors are close to the hyper plane. Like RBFNN, Support vector machines can be used for pattern classification and nonlinear regression. For linearly separable data, $S V M$ finds a separating hyper plane, which separates the data with the largest margin. 


\subsection{SVM principle}

Support Vector Machine(SVM) can be used for classifying the obtained data [15]. SVM are a set of related supervised learning method. Let us denote a feature vector by $\mathrm{x}=(\mathrm{x} 1, \mathrm{x} 2, \mathrm{xn})$ and its class lable by y such that $y=+1,-1$. Therefore, consider the problem of separating the set of n-training patterns belonging to two classes,

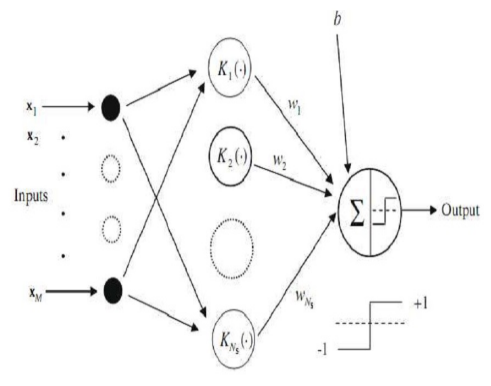

Fig. 12.

Architecture of the SVM(Ns is the number of support vectors).

$$
\left(x_{i}, y_{i}\right), x_{i} \in R^{n}, y=+1,-1, i=1,2 \ldots, n
$$

Table 2. Types of SVM inner product kernels.

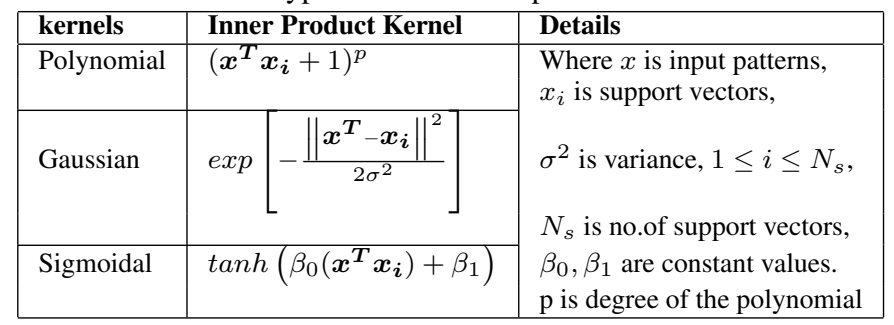

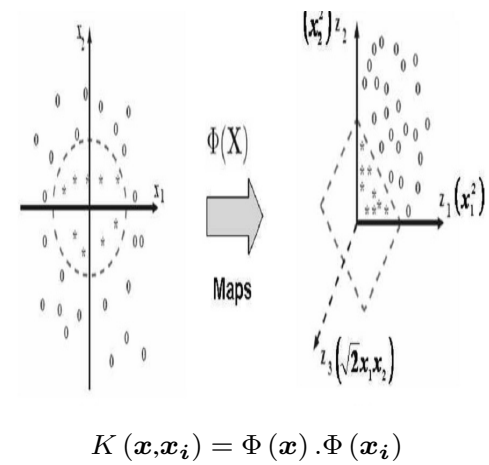

Fig. 13. An example for SVM kernel function $\Phi(\boldsymbol{x})$ maps 2-dimensional input space to higher 3-dimensional feature space. (a)Nonlinear problem. (b) Linear problem.

Given a set of images corresponding to $\mathrm{N}$ subject for training, N SVMs are trained. Each SVM was trained to distinguish between all images of a single character and all other characters in the training set. During testing, the class table $\mathrm{y}$ of a face pattern $\mathrm{x}$ can be determined using

$$
Y=\left\{\begin{array}{c}
n, \text { if } d_{n}(\boldsymbol{x})+t>0 \\
0, \text { if } d_{n}(\boldsymbol{x})+t>0
\end{array}\right\}
$$

Where, $d_{n}(X)=\operatorname{maxdi}(X)_{i}={ }^{n} 1$

The distance from $\mathrm{x}$ to the SVM hyper lance corresponding to character $i$. the classification threshold is $t$, and the class label $y=0$ stand for unknown. During testing, the distance from $x$ to the SVM hyper plane is used to identity the subject. Inner product kernel maps input space to higher dimensional feature space. Inner product kernel $\mathrm{K}(\mathrm{x}, \mathrm{X})=\phi(x), \phi(x i)$ where $\mathrm{x}$ is input patterns, $\mathrm{i}$ is support vectors.

\section{EXPERIMENTAL RESULTS}

Performance of character recognition using DCT with SVM and DWT with SVM are measured for different test samples. In this paper, we presented an image processing technique, designed for the extraction of place name region from newly introduced place name board having green colored background with white letters. The DWT features and DCT features are extracted from each character of place board and SVM is used to recognized the place name board character. Traffic place board image is captured through digital camera. The next step is to search the green pixels and convert it into black pixels using smearing algorithms. It can be observed successfully and detects the ROI that contain only place name board, it can be done by the smearing algorithm. Once the place name board is extracted, it is converted into the binary format. The horizontal and vertical segmentation methods are used to extract the individual character in the place name board. The features are extracted from individual segmented character of place name board using DCT and DWT.

\subsection{Character Recognition using DCT and SVM}

In training phase, DCT is applied to all segmented characters (A to Z) and numbers from 0 to 9 images, and features are extracted to form feature vectors in a zigzag manner or by zonal masking. These important coefficients are in from left top to zig zag manner. After applying DCT to each segmented character, 20 top highest energy coefficients are extracted, which is used as feature vector. First coefficient is a DC coefficient and the remaining 19 coefficients are AC coefficients.

\subsection{Character Recognition using DWT and SVM}

In training phase, DWT is applied to all segmented characters from $\mathrm{A}$ to $\mathrm{Z}$ and numbers from 0 to 9 images and features extracted to form feature vectors, and 324 discrete wavelet features are extracted from 36 characters $(9$ features extracted from 36 character) using DWT. In training phase, the performance of traffic place name board is evaluated for place name board image. The performance of place name board recognition using different SVM kernels with DCT and DWT are given in the table ??,

The experimental results show that the gaussian kennel gives better performance when compared to other kennels.

Figure?? shows the Place name board recognition, first one is the input image, second is the ROI detection for green, third fiqure shows the extracting place board using smearing, fourth, Converting to binary image, fifth, Line separation using row, sixth character separation using column segmentation, and seventh Recognition of Place board character using SVM. 
Table 3. Performance table

\begin{tabular}{|c|c|c|}
\hline SVM MODEL & FEATURES & RECOGNITION RATE (\%) \\
\hline gaussian & \multirow{2}{*}{ DWT } & 97.22 \\
\cline { 1 - 1 } polynomial & & 94.44 \\
\cline { 1 - 1 } sigmoidal & & 91.60 \\
\hline yaussian & \multirow{2}{*}{ DCT } & 94.44 \\
\hline polynomial & & 91.66 \\
\hline sigmoidal & & 88.80 \\
\cline { 1 - 1 } & &
\end{tabular}

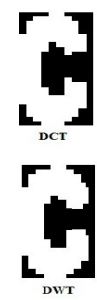

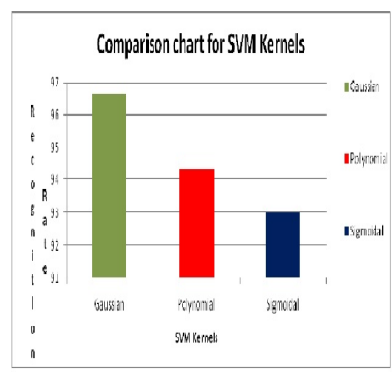

Fig. 15. Comparison chart for SVM kernnels

\section{CONCLUSION}

We present an algorithm for automatic place board character recognition from natural scenes. We propose an approach to detect, segment, feature extract and recognize the characters appearing in color image and gray scale images. It detect the green colored signage's from the background using seamearing algorithm and extract the detected signage's board then convert it into binary image. Next, it detect the signage characters from signage's using horizontal segmentation and vertical segmentation algorithms, it extract each individual segmented character from the signage's. Then, the features are extracted from individual segmented character of signage's using DCT and DWT. In training phase, 324 discrete wavelet features are extracted from 36 characters $(9$ features extracted from 36 character) in DWT and 20 highest energy coefficients are extracted by using DCT. Finally, the extracted features from each characters are recognized using SVM. The algorithm framework considers critical challenges in text detection and segmentation robustly in different conditions and different backgrounds. The proposed horizontal and vertical segmentation proves to extract text region efficiently. The contribution is to apply DCT, DWT and SVM efficiently.

\section{REFERENCES}

[1] V.N. Manjunath Aadha, M.S. Pavithra, C. Naveena, A Robust Multilingual Detection approach based on transvele entropy, Elsevier-C3IT, 4, 232-237(2012)

[2] Q.Ye, Q. Huang,W.Gao,D.Zhao,Fast and robust text detection in images and video frames,Image and vision computing, 23 , 565-576(2005)

[3] D.Chen, J.Odobez, H.Bourlard, Text Detection and recognition in images video frames, Pattern recognition, 37, 595-608, 2004.

[4] R.Lienhart and A.Wernicke, Localising and segmenting text in images and videos, IEEE transactions on circuits and systems for video technology, $12,2002$.

[5] Jianqiang Yan, Jie Li, Xinbo Gao, Chinese text location under complex background using gabor filter and SVM, neuro computing, 74, 2998-3008, 2011.

[6] L Xu, A.Krzyzak, C.Y.Suen, Methods of combining multiple classifier and their applications to handwritting recognition, IEEE transaction on system Man and cybernetics, 27, 418-435, 1992.

[7] J.Kittler, M.Hatef, R.P.W.Duin, J.Matas, On combining classifier, IEEE transaction on pattern analysis and machine intelligence, 20, 226-239, 1998.

[8] Hemant Misra, Francois Yvon, Olivier cappe, Joemon Jose, Text segmentation: A topic modelling perspective, Information processing and management ,47,528-544,2011.

[9] Detection from natural scenes by structure-based partition and grouping, IEEE transaction on Image processing, 20, 2011.

[10] Xiaodong Huang, Huadong Ma Beijing, Automatic detection and localiz tion of natural scene text in video ,IEEE-pattern recognition(CIPR),3216-3219,2010.

[11] Blei, D.M.,and Moreno,Topic segmentation with an aspect HMM,ACM-special interest group on information retrival(SIGIR),343-348,2001

[12] V.Vapnik, Statistical Learning Theory, John Wiley and sons,newyork, 1998.

[13] J.C.Burges Christopher, A tutorial on support vector machine for pattern recognition, 52,121-167,1998.

[14] Suchitra Shrestha and Khan Wahid, Hybrid DWT-DCT Algorithm for Biomedical Image and Video Compression Applications, Proc. of the 10th IEEE International Conference on Information Sciences, Signal Processing and their Applications, pp. 280-283, 2010.

[15] Suchitra Shrestha and Khan A Wahid, A sub-sample based hybrid DWT-DCT algorithm for medical imaging applications, Cyber Journals: Multidisciplinary Journals in Science and Technology, Nov. 24, 2010. 